\title{
QTLs for agronomic traits detected in recombinant inbred lines derived from a
}

\section{bread wheat $\times$ spelt cross}

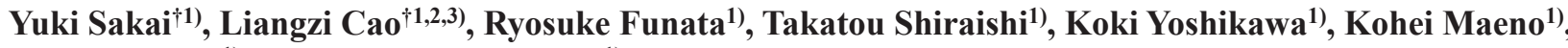 \\ Hideho Miura $^{1)}$ and Kazumitsu Onishi*1) \\ 1) Obihiro University of Agriculture and Veterinary Medicine, Hokkaido 080-8555, Japan \\ 2) The United Graduate School of Agricultural Sciences, Iwate University, Iwate 020-8550, Japan \\ 3) Institute of Crop Cultivation and Tillage, Heilongjiang Academy of Agricultural Sciences, Heilongjiang 150086, China
}

\begin{abstract}
Spelt wheat (Triticum aestivum subsp. spelta), a subspecies of common wheat, is a genetic resource for the breeding of bread wheat (T. aestivum subsp. aestivum); however, genetic analyses of agronomic traits in bread wheat $\times$ spelt crosses are insufficient. Here, we conducted QTL analysis in the recombinant inbred lines from a bread wheat $\times$ spelt cross. In addition to the major $Q$ locus, $Q S p d . o b u-4 D$ was detected with the spelt allele conferring a higher spikelet density than the bread wheat allele. The effect of $Q S p d . o b u-4 D$ was evident in the presence of the $Q$ allele of bread wheat, suggesting that this variation might be cryptic in spelt wheat with the $q$ allele. Two QTLs with stable effects were identified for grain length, one of which (QGl.obu- $1 A)$ has never been detected in a bread wheat $\times$ spelt cross. The spelt wheat allele at QHt.obu-7B conferring later heading was identified in the $V r n-B 3$ region and could be a novel gene source for modifying heading time. Furthermore, QGi.obu-2B, responsible for low grain dormancy of spelt wheat, was detected. Further exploration and identification of useful QTLs could accelerate the utilization of spelt wheat as a genetic resource for bread wheat breeding programs.
\end{abstract}

Key Words: grain dormancy, grain morphology, heading time, QTL analysis, spelt wheat, spike morphology.

\section{Introduction}

Wheat has been an important cereal crop since the ancient times and is cultivated under various climatic conditions around the world. Approximately $95 \%$ of wheat cultivated today is bread wheat (Triticum aestivum subsp. aestivum), although wheat comprises a variety of species and subspecies (Matsuoka 2011). Bread wheat is a hexaploid wheat species, with AABBDD genomes, and shows the free threshing trait. In contrast, spelt wheat (T. aestivum subsp. spelta) shows hulled or non-free threshing traits, although it is considered as one of the subspecies of common wheat and carries AABBDD genomes. Spelt and bread wheat are closely related and can produce fertile descendants via hybridization, although spelt wheat harbors distinct genetic variations in its genome (Kippes et al. 2015). Even though spelt wheat has only minor economic importance at present,

Communicated by Kenji Kato

Received April 10, 2018. Accepted September 6, 2018.

First Published Online in J-STAGE on November 28, 2018.

*Corresponding author (e-mail: onishi@obihiro.ac.jp)

${ }^{\dagger}$ These authors contributed equally to this work it has potential as a genetic resource for the breeding of bread wheat, because of its superior genetic variation for traits such as yield and yield components, disease resistance, and abiotic stress tolerance (Campbell 1997, Raman et al. 2009, Xie et al. 2015a). However, spelt wheat also has many undesirable traits, including lower yield, higher plant height, and increased lodging, compared to the modern bread wheat varieties, which are disadvantageous in the modern agricultural system (Miedaner and Longin 2016).

Spelt wheat is best distinguished from bread wheat by its non-free threshing trait and the long and lax spike morphology. In spelt wheat, the glumes tenaciously enclose grains and a strong mechanical force is required to separate the grains from the glumes. The differences in spike morphology and threshability are largely controlled by the $Q$ locus on chromosome 5A, with spelt and bread wheat carrying the $q$ and $Q$ alleles, respectively (Simons et al. 2006). The $q$ allele of the spelt wheat confers the non-free threshing trait and lax spike morphology (speltoid type), whereas the $Q$ allele of the bread wheat confers the free-threshing trait and spike morphology of the square head type (Simons et al. 2006). The non-free threshing trait and the speltoid type spike prevent efficient harvesting and could impact the yield; 
therefore, the $q$ allele should preferably be eliminated from current bread wheat breeding programs. In addition to the effects of the $Q$ locus, a wide range of variation in spike morphology, such as spike length and spikelet density has been reported in hybrid populations between spelt and bread wheat (Dvorak et al. 2012, Manickavelu et al. 2011, Onishi et al. 2006, Xie et al. 2015a). Therefore, understanding the genetic control of spike morphology, which is closely related to yield and other yield components, is necessary for the utilization of spelt wheat in bread wheat breeding programs.

Grain morphology is associated with yield, processing properties, and end-use quality; therefore, it is an important agronomic trait for wheat. Spelt wheat tends to have longer and larger grains than bread wheat (Gegas et al. 2010), although bread wheat displays a lot of variations. Heading time and pre-harvest sprouting (PHS) tolerance are also important agronomic traits for adaptation to local environments in diverse regions. The degree of grain dormancy is considered to be the most critical factor for PHS tolerance and could be important especially in wet and humid climatic regions, such as Japan, because PHS is a major problem affecting grain quality. A number of QTLs for grain morphology, heading time and grain dormancy have been reported in bread wheat (Kamran et al. 2014, Mares and Mrva 2014), while there have been only a few studies involving the genetic analysis of spelt wheat (Liu et al. 2017, Manickavelu et al. 2011, Xie et al. 2015a, Zanetti et al. 2000).

To use spelt wheat as a breeding material, it is necessary to compare the QTLs responsible for agronomic traits detected in different populations derived from the crosses using diverse spelt germplasm as wide genetic variation in spelt wheat may exist. However, to date, only a few populations have been used in genome-wide QTL analyses (Liu et al. 2017, Manickavelu et al. 2011, Xie et al. 2015a). In the present study, we developed new recombinant inbred lines from a cross between spelt and bread wheat and conducted QTL analysis for agronomic traits, including spike and grain morphology, heading time, and grain dormancy.

\section{Materials and Methods}

\section{Plant materials}

The plant materials used in this study were recombinant inbred lines (RILs) developed from the $\mathrm{F}_{2}$ population of a cross between 'Chinese Spring' (CS) and a spelt wheat strain, 'st. Rumania' (RU), by the single-seed descent method. RU was the strain collected in Romania and provided by the National BioResource Project-Wheat (Strain ID: KT019-002), with support, in part, from the National BioResource Project of the Ministry of Education, Culture, Sports, Science \& Technology (MEXT), Japan. Ninety-six RILs from $\mathrm{F}_{9}$ or later generations were used for the phenotypic evaluation and linkage map construction.

\section{Cultivation}

The RILs and their parental strains (CS and RU) were cultivated under three environmental conditions at the Obihiro University of Agriculture and Veterinary Medicine, in Obihiro, Japan.

In the experiments conducted in 2014 (designated as 2014), RILs and their parental strains were grown in the field. Seeds were sown in cell flats in late April. After approximately three weeks, the seedlings were transplanted in rows of $40 \mathrm{~cm}$ length $(10 \mathrm{~cm}$ between plants and $30 \mathrm{~cm}$ between rows) in the experimental field covered with a transparent plastic roof to prevent damages from rain. Four plants per row were grown with one replication (row) for each RIL and with nine replications (rows) of parental strains. Eighteen of 36 plants were used for phenotypic evaluation of the parental strains. Fertilizer $\left(\mathrm{N}, 10 \mathrm{~g} ; \mathrm{P}_{2} \mathrm{O}_{5}\right.$, $15 \mathrm{~g} ; \mathrm{K}_{2} \mathrm{O}, 9 \mathrm{~g} \mathrm{~m}^{-2}$ ) was applied before transplanting.

In the experiments conducted in 2015 (designated as $2015)$, seeds were sown in plastic boxes $(60 \times 15 \mathrm{~cm}, 81$ volume) in late April. The seedlings were grown for approximately three weeks in a greenhouse and then the plastic boxes were placed outside. Eight plants per box were grown for each RIL with one replication (box) and with five replications (boxes) for parental strains. After anthesis, the plastic boxes were moved under a plastic roof to prevent damages from rain. Four of eight plants for each RIL and 20 of 40 plants of the parental strains were used in the experiments. To eliminate variability due to soil factors, soil from the field was mixed well and apportioned to all plastic boxes. Fertilizer (N, $0.5 \mathrm{~g} ; \mathrm{P}_{2} \mathrm{O}_{5}, 1.0 \mathrm{~g} ; \mathrm{K}_{2} \mathrm{O}, 0.5 \mathrm{~g}$ per box) was applied for each plastic box before seed sowing.

In the experiment conducted in 2016 (designated as 2016), the RILs were grown in a controlled environment to maintain constant temperature during the growth period, because variations in heading time triggers the RILs to develop spikes and grains under different temperature conditions. In addition, RILs were grown after vernalization because the range of heading time in the RILs was narrower post-vernalization than without. Seeds were sown in cell flats and grown for 10 days in an incubator (LH-350SP, Nippon Medical \& Chemical Instruments Co., Ltd., Osaka, Japan) under an $8 \mathrm{~h}$ day-length and at $23^{\circ} \mathrm{C}$ day $/ 18^{\circ} \mathrm{C}$ night temperature. Then, the seedlings were vernalized $(8 \mathrm{~h}$ daylength and $5^{\circ} \mathrm{C}$ day and night temperature) for 30 days in the incubator. After vernalization, the seedlings were transplanted into pots (13 cm diameter, 11 volume) and grown under controlled-temperature conditions with natural sunlight $\left(22^{\circ} \mathrm{C}, 12 \mathrm{~h}\right.$ day $/ 16^{\circ} \mathrm{C}, 12 \mathrm{~h}$ night). Two plants for each RIL and eight plants for each of the parental strains were grown. Fertilizer $\left(\mathrm{N}, 0.05 \mathrm{~g} ; \mathrm{P}_{2} \mathrm{O}_{5}, 0.1 \mathrm{~g} ; \mathrm{K}_{2} \mathrm{O}, 0.05\right.$ g per pot) was applied to each pot before transplanting.

\section{Phenotypic evaluation}

Spike and grain morphology and heading time (HT) were measured in all $(2014,2015$, and 2016) the experiments. Spikes were harvested after maturity and were stored at room temperature (approximately $25^{\circ} \mathrm{C}$ ) until measurement. Spike length (SPL) was measured from the base of the spike 
to the tip of the top-most spikelet excluding the awn. Number of spikelets (SPN) was counted and spikelet density (SPD) was calculated as SPN/SPL. After hand-threshing, grain length (GL) and width (GW) were measured using SmartGrain (Tanabata et al. 2012). Grain thickness was measured using Vernier calipers. Two to four spikes and 10 to 40 grains were measured for each RIL. Eight to 20 spikes and 60 to 180 grains were measured for each parental strain.

Germination tests were conducted to evaluate the degree of grain dormancy in the 2014 and 2015 experiments. Germination tests could not be conducted in 2016 due to limited harvest material. Each spike was checked daily and harvested at physiological maturity (when the green color of the spike disappeared completely). Spikes were air-dried at room temperature (approximately $25^{\circ} \mathrm{C}$ ) for approximately three days and were preserved at $-20^{\circ} \mathrm{C}$ in a freezer until use. Two to six spikes of RILs and six to ten spikes for the parental strains were harvested. Grains were carefully separated from the glumes by hand-threshing and were placed on a filter paper wetted with water in petri dishes $(90 \mathrm{~mm}$ diameter). The dishes were incubated at $20^{\circ} \mathrm{C}$ for 10 days in the dark. The number of germinated grains was counted daily. When the seed coat ruptured, the grain was considered to have germinated. The degree of grain dormancy was calculated as the germination index (GI), which was: GI = $\left(10 \times s_{1}+9 \times s_{2}+\ldots+1 \times s_{10}\right) /($ total number of grains examined $\times 10$ ), where $s_{n}$ is the number of grains that germinated on the $n^{\text {th }}$ day (Mohan et al. 2009).

\section{Marker analysis and linkage map construction}

Total DNA from each RIL was extracted as described by Monna et al. (2002) and with slight modifications as per Cao et al. (2016). Briefly, small pieces of young leaves were sampled in a 2-ml tube. Leaves were frozen using liquid nitrogen and crushed using stainless steel beads. Approximately $500 \mu$ of DNA extraction buffer, as per Monna et al. (2002), was added. After $15 \mathrm{~min}, 400 \mu \mathrm{l}$ of chloroform:isoamyl alcohol (24:1) was added and mixed with the solution by inversion. After mixing, the tube was spun at 13,000 rpm for $15 \mathrm{~min}$ and the supernatant was collected in a new tube. Isopropanol was added to the tube and centrifuged again at 13,000 rpm for $15 \mathrm{~min}$ to precipitate the crude DNA. The DNA pellet was rinsed with $70 \%$ ethanol and dissolved in Tris-EDTA Buffer.

Amplification of genetic markers was performed by polymerase chain reaction (PCR) and using GoTaq ${ }^{\circledR}$ DNA polymerase (Promega Corporation, Madison, WI, USA) or KOD FX and KOD FX Neo DNA polymerase (TOYOBO CO., LTD., Osaka, Japan) or PrimeSTAR ${ }^{\circledR}$ GXL DNA polymerase (Takara Bio Inc., Shiga, Japan), according to the manufacturer's protocols. The PCR products were electrophoresed in 3-4\% agarose gels or $10 \%$ acrylamide gels (acrylamide: $\quad N, N^{\prime}$-methylenebisacrylamide $=29: 1$ ). The genotype bands were evaluated after staining with ethidium bromide.

For the linkage map construction, 160 simple sequence repeat (SSR) markers and four gene-specific markers were used. The SSR markers were selected based on the information from Somers et al. (2004) and GrainGenes (https:// wheat.pw.usda.gov/GG3/) (Supplemental Table 1). The four gene-specific markers of the $Q$ (Asakura et al. 2009), Vrn-A1 (Yan et al. 2004), Vrn-D1 (Fu et al. 2005), and ALMT-1 loci (Sasaki et al. 2006), which were polymorphic between the parental strains were used for map construction. Based on the genotypes of 96 RILs, the linkage map was constructed using JoinMap ${ }^{\circledR} 4$ software (van Ooijen 2006).

\section{Data analysis}

The MQM (multiple QTL model) mapping method was used for QTL analysis by using MapQTL ${ }^{\circledR} 6$ (van Ooijen 2002). In the first step, interval mapping (IM) was performed to identify the putative QTLs. one marker at each putative QTL (with LOD greater than two) detected by IM was selected as a cofactor and multiple-quantitative trait loci model (MQM) mapping was performed. The markers closest to the significant QTL were finally selected as a set of cofactors. The genome-wide LOD threshold at the 5\% level were determined using a permutation test for each trait (Churchill and Doerge 1994).

To analyze the interactions between the two QTLs for spikelet density, the differences between the genotypes were tested using the Tukey-Kramer test for multiple comparisons using PASW ${ }^{\circledR}$ statistics 18 (SPSS Inc., Chicago, IL, USA).

\section{Results}

\section{Genetic variation in agronomic traits in RILs}

The spike and grain morphology in RILs and their parental strains was examined under three environmental conditions (Table 1). The spelt wheat strain (RU) consistently showed longer SPL $(13.1-13.9 \mathrm{~cm})$ and lower SPD (1.3 spikelets $/ \mathrm{cm})$ than the bread wheat strain (CS) $(6.9$ $8.0 \mathrm{~cm}$ in SPL, 2.5-2.6 spikelets/cm in SPD). Differences in SPN between CS and RU were small (0.5-2.1), whereas they were significant only in $2015(p<0.001)$ and 2016 $(p=0.015)$. In the RILs, there was large transgressive segregation in SPL, SPN, and SPD, and the lines with shorter SPL and greater SPD than CS were appeared in RILs (Table 1, Fig. 1A). The GL of RU (7.6-7.8 mm) was greater than that of CS $(5.7-6.1 \mathrm{~mm})$ and there were only small differences (within $0.2 \mathrm{~mm}$ ) in GW between the two strains (Table 1, Fig. 1B). No significant differences were found with respect to GT. RILs showed continuous variation of GL between the values for parental strains. Transgressive segregation was observed for GW and GT.

The difference of HT between CS and RU in 2014 and 2015 without vernalization was small (1.8 and 1.3 days, respectively) but it increased ( 8.9 days) after vernalization in 2016 (Table 1). Transgressive segregation towards later HT was observed in all the experiments. The GI of RU was 
Table 1. Comparison of spike and grain related traits in parental strains and RILs

\begin{tabular}{|c|c|c|c|c|c|c|c|}
\hline \multirow{2}{*}{\multicolumn{2}{|c|}{ Trait }} & \multirow{2}{*}{ Trial } & \multirow{2}{*}{$\mathrm{CS}$} & \multirow{2}{*}{$\mathrm{RU}$} & \multirow{2}{*}{$t$-test } & \multicolumn{2}{|c|}{ RILs } \\
\hline & & & & & & Mean & Range \\
\hline \multirow{3}{*}{\multicolumn{2}{|c|}{$\mathrm{SPL} \quad(\mathrm{cm})$}} & 2014 & 7.3 & 13.8 & $* * *$ & 9.3 & $4.1-14.8$ \\
\hline & & 2015 & 8.0 & 13.9 & $* * *$ & 9.4 & $4.8-14.1$ \\
\hline & & 2016 & 6.9 & 13.1 & $* * *$ & 9.5 & $4.7-14.5$ \\
\hline \multirow{3}{*}{\multicolumn{2}{|c|}{ SPN }} & 2014 & 18.1 & 18.6 & $\mathrm{~ns}$ & 17.1 & $12.3-22.3$ \\
\hline & & 2015 & 20.5 & 18.4 & $* * *$ & 19.0 & $15.0-24.8$ \\
\hline & & 2016 & 18.3 & 17.0 & $*$ & 17.5 & $13.5-21.0$ \\
\hline \multirow[t]{3}{*}{ SPD } & (spikelets/cm) & 2014 & 2.5 & 1.3 & $* * *$ & 2.0 & $1.1-3.5$ \\
\hline & & 2015 & 2.6 & 1.3 & $* * *$ & 2.2 & $1.3-4.2$ \\
\hline & & 2016 & 2.6 & 1.3 & $* * *$ & 2.0 & $1.0-3.6$ \\
\hline \multirow[t]{3}{*}{ GL } & $(\mathrm{mm})$ & 2014 & 5.7 & 7.7 & $* * *$ & 6.6 & $5.6-7.6$ \\
\hline & & 2015 & 5.7 & 7.6 & $* * *$ & 6.7 & $5.4-7.8$ \\
\hline & & 2016 & 6.1 & 7.8 & $* * *$ & 7.1 & $5.9-8.3$ \\
\hline \multirow[t]{3}{*}{ GW } & $(\mathrm{mm})$ & 2014 & 3.0 & 3.0 & $\mathrm{~ns}$ & 3.0 & $2.4-3.2$ \\
\hline & & 2015 & 2.7 & 2.9 & $* *$ & 2.9 & $2.6-3.3$ \\
\hline & & 2016 & 3.1 & 3.0 & $*$ & 3.2 & $2.7-3.4$ \\
\hline \multirow[t]{3}{*}{ GT } & $(\mathrm{mm})$ & 2014 & 2.6 & 2.6 & ns & 2.7 & $2.3-3.0$ \\
\hline & & 2015 & 2.5 & 2.7 & ns & 2.7 & $2.3-3.2$ \\
\hline & & 2016 & 2.9 & 2.9 & ns & 3.0 & $2.5-3.5$ \\
\hline \multirow[t]{3}{*}{ HT } & (days) & 2014 & 63.7 & 65.5 & $*$ & 68.8 & $61.3-86.0$ \\
\hline & & 2015 & 61.8 & 60.5 & $\mathrm{~ns}$ & 65.7 & $55.5-82.5$ \\
\hline & & 2016 & 73.4 & 82.3 & $* * *$ & 81.7 & $74.0-92.0$ \\
\hline \multirow[t]{2}{*}{ GI } & & 2014 & 0.52 & 1.00 & $* * *$ & 0.68 & $0.04-1.00$ \\
\hline & & 2015 & 0.37 & 0.94 & $* * *$ & 0.51 & $0.00-1.00$ \\
\hline
\end{tabular}

GL: Grain length, GW: Grain width, GT: Grain thickness, SPL: Spike length, SPN: Spikelet numbers per spike, SPD: Spikelet density (SPN/ SPL), HT: Heading time, GI: Germination index. *, ** and *** indicate that the significance at the 5,1 and $0.1 \%$ levels respectively. ns indicates non-significance.
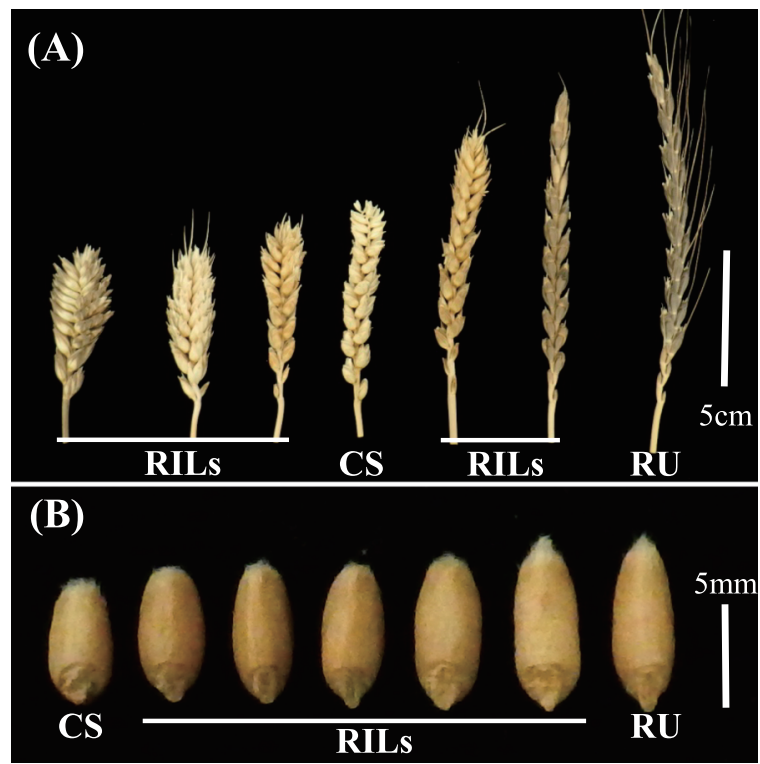

Fig. 1. Variation in spike (A) and grain (B) morphologies observed in the parental strains and RILs.

significantly higher than that of CS (Table 1). The GI of RU was nearly 1.0 , indicating that most grains germinated on the first and second day because of a very low level of grain dormancy. Continuous distributions of GI were observed in the RILs.

\section{QTL analysis}

A genetic linkage map was constructed using 164 molecular markers (Fig. 2). The total map length was $1,902 \mathrm{cM}$ and it covered the majority of the wheat genome compared to a consensus map of 2,569 cM as reported in Somers et al. (2004), although markers were scarce in some chromosomal regions.

QTL analysis was conducted for eight examined traits and significant QTLs were detected for all traits under at least one environmental condition (Fig. 2, Table 2). For spike morphology, QTLs were detected on chromosomes 4D and 5A. The QTLs on chromosome 5A (QSpl.obu-5A and $Q S p d . o b u-5 A$ ) were detected in the region including the $Q$ locus and had strong effects that increased SPL and decreased SPD due to the RU-derived allele. The effects of QSpl.obu-5A and QSpd.obu-5A were consistent with the expected genetic effects of the $Q$ locus, a major genetic locus that controls spike morphology and threshability (Simons et al. 2006). In contrast, QTLs on chromosome 4D (QSpl.obu-4D and QSpd.obu-4D) had effects that decreased SPL and increased SPD due to the RU-derived allele, although the magnitude of these effects was smaller than QSpl.obu-5A and QSpd.obu-5A.QSpd.obu-4D was detected in all three environmental conditions.

QTLs with stable effects on grain morphology under all environmental conditions were detected on chromosomes 1A (QGl.obu-1A) and 2D (QGl.obu-2D) for GL, and near the $Q$ locus on chromosome 5A for GT (QGt.obu-5A) (Fig. 2, Table 2). The RU-derived alleles at QGl.obu- $1 \mathrm{~A}$ and $Q G$ l.obu-2D increased GL, while the CS-derived alleles at QGt.obu-5A increased GT. QTLs for GL on chromosomes $3 \mathrm{~A}$ and $4 \mathrm{D}$ and those for $\mathrm{GW}$ on chromosomes $2 \mathrm{~B}$ and $5 \mathrm{~A}$ were significant under only one or two environmental conditions.

For HT, three QTLs were found on chromosomes 2B (QHt.obu-2B), 5A (QHt.obu-5A) and 7B (QHt.obu-7B) (Fig. 2, Table 2). The QTL QHt.obu-5A had the largest effect (37.9 and 54.6\% in PVE) in 2014 and 2015 without vernalization, while it could not be detected in 2016 with vernalization. In contrast, the QTL QHt.obu- $2 B$ was only visible in the 2016 experiments and required vernalization. The QTL QHt.obu-7B had stable effects in conferring later heading due to the RU-derived allele in all environments with or without vernalization. Most QTLs for spike and grain morphology were independent to QTLs for HT, except that a number of QTLs were tightly linked in the $Q$ locusQHt.obu-5A region.

For GI, a significant QTL (QGi.obu-2B) was detected on chromosome $2 \mathrm{~B}$ in two environmental conditions examined (Fig. 2, Table 2). The RU-derived allele increased the GI (decreased the level of grain dormancy). Although QGi.obu$2 B$ and QHt.obu-2B were co-located on chromosome 2B, these two QTLs were detected independently in different environments. 

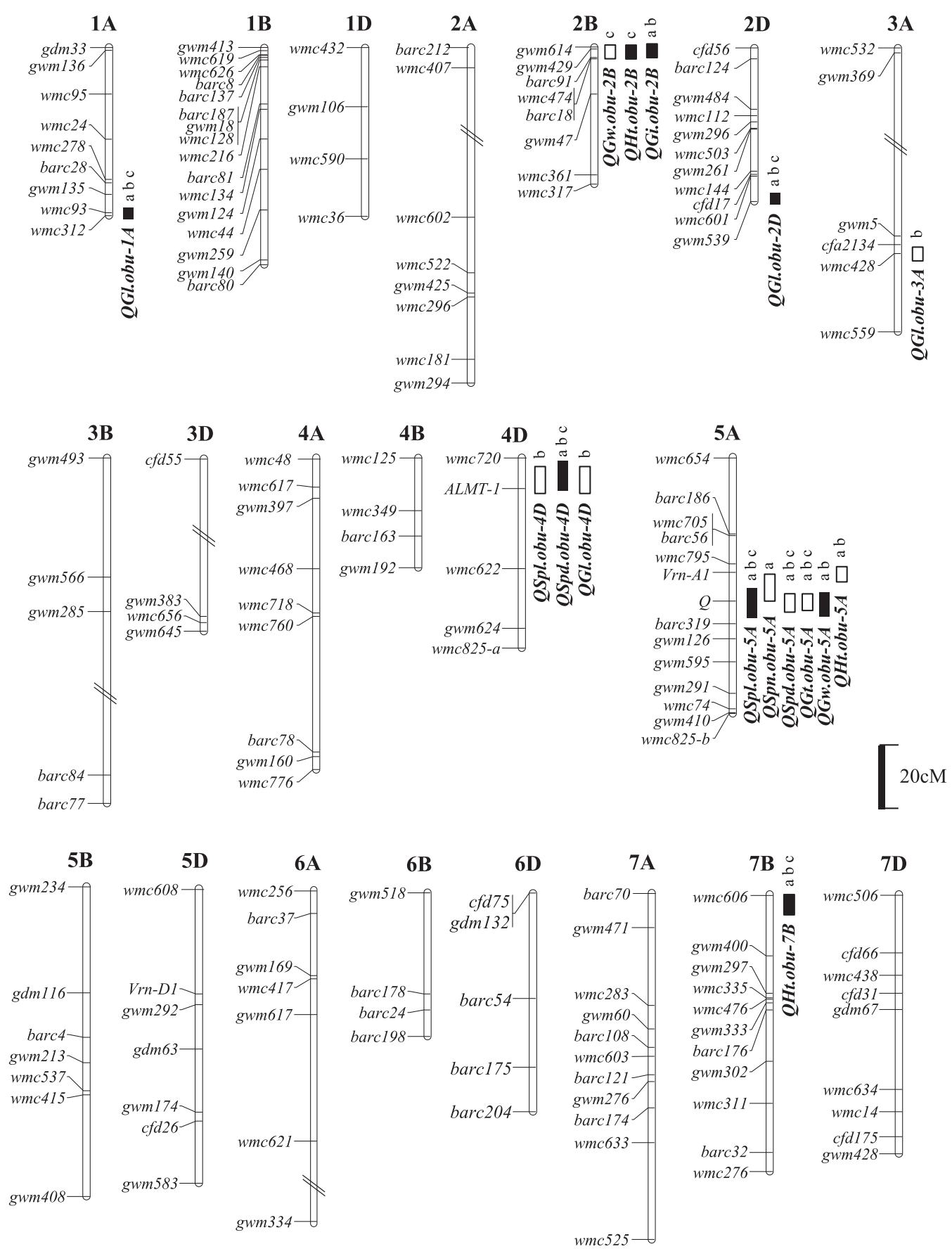

Fig. 2. Linkage map indicating the positions of QTLs detected in the RILs. Markers used for map construction are shown on the left side of the chromosomes. Locations of QTLs detected by MQM mapping are represented as boxes with two-LOD support intervals. Filled and white boxes indicate the effects of the QTLs that increase the trait values by the RU- and CS-derived alleles, respectively. a, b, and c indicate QTLs detected in 2014, 2015, and 2016, respectively.

\section{Interaction of QTLs for spikelet density}

Conspicuous transgressive segregation was observed for SPD, in which a compact spike appeared in the RILs (Fig. 1A). A QTL for SPD on chromosome 4D (QSpd.obu$4 D$ ) led to an increase in SPD through the RU-derived allele, although RU normally showed a lax spike morphology. To understand the genetic mechanism of transgressive segregation, the interaction between QTLs on chromosomes $5 \mathrm{~A}(Q$ locus) and 4D (QSpd.obu-4D) was examined (Fig. 3). The RILs were classified into four genotype classes based on the allele combinations of the two loci. Under the presence of the $q$ allele derived from RU, $Q S p d . o b u-4 D$ had no effect on SPD; however, the RU-derived alleles at QSpd.obu-4D caused significant increases in SPD in combination with the $Q$ allele derived from CS, indicating that the $q$ allele suppressed the effect of the RU-derived alleles at QSpd.obu- $4 D$. 
Table 2. Results of MQM mapping for spike and grain related traits in the RILs

\begin{tabular}{|c|c|c|c|c|c|c|}
\hline Trait & Trial & $\begin{array}{l}\text { Chromo- } \\
\text { some }\end{array}$ & QTL & LOD & PVE (\%) & $\begin{array}{c}\text { Additive } \\
\text { effect }\end{array}$ \\
\hline \multirow[t]{4}{*}{ SPL } & 2015 & 4D & QSpl.obu-4D & 3.5 & 6.0 & 7.0 \\
\hline & 2014 & $5 \mathrm{~A}$ & QSpl.obu-5A & 20.5 & 63.8 & -2.3 \\
\hline & 2015 & $5 \mathrm{~A}$ & QSpl.obu-5A & 21.9 & 63.8 & -2.1 \\
\hline & 2016 & $5 \mathrm{~A}$ & QSpl.obu-5A & 29.7 & 75.9 & -2.6 \\
\hline SPN & 2014 & $5 \mathrm{~A}$ & QSpn.obu-5A & 5.3 & 23.0 & 0.9 \\
\hline \multirow[t]{6}{*}{ SPD } & 2014 & $4 \mathrm{D}$ & QSpd.obu-4D & 4.6 & 7.6 & -0.2 \\
\hline & 2015 & $4 \mathrm{D}$ & QSpd.obu-4D & 4.9 & 9.2 & -0.2 \\
\hline & 2016 & $4 \mathrm{D}$ & QSpd.obu-4D & 3.3 & 5.3 & -0.2 \\
\hline & 2014 & $5 \mathrm{~A}$ & QSpd.obu-5A & 22.0 & 56.0 & 0.6 \\
\hline & 2015 & $5 \mathrm{~A}$ & QSpd.obu-5A & 18.0 & 50.1 & 0.5 \\
\hline & 2016 & $5 \mathrm{~A}$ & QSpd.obu-5A & 21.4 & 56.0 & 0.5 \\
\hline \multirow[t]{8}{*}{ GL } & 2014 & $1 \mathrm{~A}$ & QGl.obu-1A & 2.9 & 9.9 & -0.1 \\
\hline & 2015 & $1 \mathrm{~A}$ & QGl.obu-1A & 3.1 & 6.7 & -0.1 \\
\hline & 2016 & $1 \mathrm{~A}$ & QGl.obu-1A & 4.4 & 16.2 & -0.2 \\
\hline & 2014 & $2 \mathrm{D}$ & QGl.obu-2D & 5.4 & 19.3 & -0.2 \\
\hline & 2015 & $2 \mathrm{D}$ & QGl.obu-2D & 5.0 & 11.3 & -0.2 \\
\hline & 2016 & $2 \mathrm{D}$ & QGl.obu-2D & 2.9 & 10.3 & -0.2 \\
\hline & 2015 & $3 \mathrm{~A}$ & QGl.obu-3A & 3.5 & 7.7 & 0.1 \\
\hline & 2015 & $4 \mathrm{D}$ & QGl.obu-4D & 3.9 & 8.5 & 0.1 \\
\hline \multirow[t]{3}{*}{ GW } & 2016 & $2 \mathrm{~B}$ & $Q G w . o b u-2 B$ & 5.9 & 24.7 & 0.1 \\
\hline & 2014 & $5 \mathrm{~A}$ & $Q G w . o b u-5 A$ & 3.0 & 13.7 & -0.1 \\
\hline & 2015 & $5 \mathrm{~A}$ & $Q G w . o b u-5 A$ & 4.6 & 20.1 & -0.1 \\
\hline \multirow[t]{3}{*}{ GT } & 2014 & $5 \mathrm{~A}$ & QGt.obu-5A & 5.4 & 23.4 & 0.1 \\
\hline & 2015 & $5 \mathrm{~A}$ & QGt.obu-5A & 8.4 & 33.3 & 0.1 \\
\hline & 2016 & $5 \mathrm{~A}$ & QGt.obu-5A & 7.8 & 31.0 & 0.1 \\
\hline \multirow[t]{6}{*}{ HT } & 2016 & $2 \mathrm{~B}$ & QHt.obu-2B & 6.7 & 25.3 & -2.0 \\
\hline & 2014 & $5 \mathrm{~A}$ & QHt.obu-5A & 10.5 & 37.9 & 3.5 \\
\hline & 2015 & $5 \mathrm{~A}$ & QHt.obu-5A & 17.3 & 54.6 & 5.6 \\
\hline & 2014 & $7 \mathrm{~B}$ & QHt.obu-7B & 4.0 & 12.7 & -2.2 \\
\hline & 2015 & $7 \mathrm{~B}$ & QHt.obu-7B & 4.5 & 9.9 & -2.7 \\
\hline & 2016 & $7 \mathrm{~B}$ & QHt.obu-7B & 3.8 & 12.6 & -1.8 \\
\hline \multirow[t]{2}{*}{ GI } & 2014 & $2 \mathrm{~B}$ & QGi.obu-2B & 3.1 & 14.6 & -0.10 \\
\hline & 2015 & $2 \mathrm{~B}$ & QGi.obu-2B & 3.8 & 19.4 & -0.12 \\
\hline
\end{tabular}

LOD values, PVE (phenotypic variance explained by each QTL), and additive effect are listed. Positive and negative values of additive effect indicate the effect increasing trait values by the CS and RU alleles, respectively.

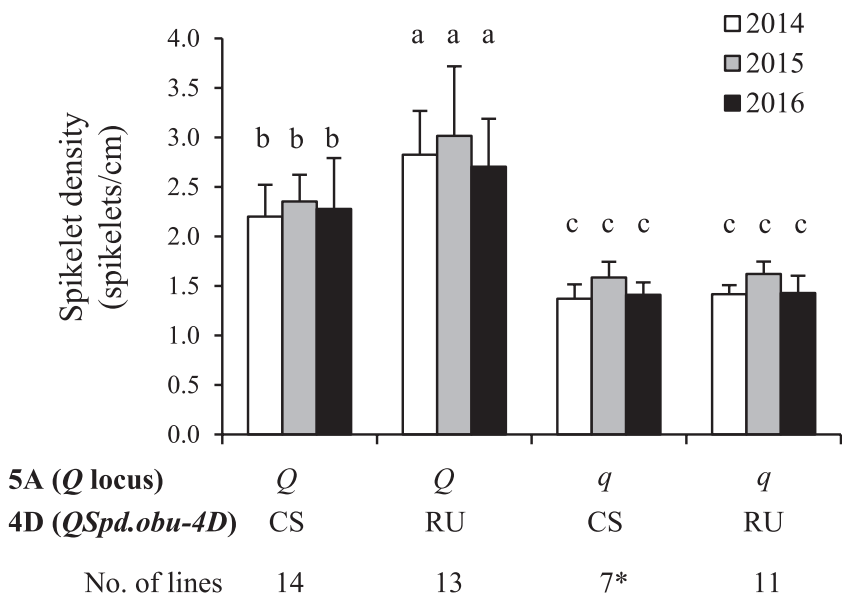

Fig. 3. Interaction of two QTLs for spikelet density on chromosomes 5A (the $Q$ locus) and 4D (QSpd.obu-4D). Two markers (wmc720 and $A L M T-1)$ or three markers (barc319, $Q$, and $V r n-A 1)$ were used to determine the genotype of QTL on chromosomes 4D and 5A, respectively. Different letters on the bars indicate significant differences between genotypes in the same experiment. *: Six lines were used in 2015.

\section{Comparison to known QTLs or genes}

The QTLs with stable effects for spike and grain morphology detected in the present study (QGl.obu-1A, QGl.obu$2 D$ and $Q S p d . o b u-4 D$ ) were compared with the previously reported QTLs in populations derived from crosses between spelt and bread wheat (Fig. 4) (Manickavelu et al. 2011, Xie et al. 2015a, 2015b). The QTL QGl.obu-2D was consistent with the region detected in Manickavelu et al. (2011), in which the same parent strain of bread wheat (CS) was crossed with a different spelt wheat strain. The $T g-D 1$ gene for non-free threshing on chromosome 2D has pleiotropic effects on increasing grain length (Okamoto et al. 2012); the location of $T g-D 1$ is different from that of $Q G l . o b u-2 D$, as this QTL was mapped at the terminal region of the long arm of the chromosome. Manickavelu et al. (2011) also identified a QTL for SPD (QCpt) near QSpd.obu-4D on chromosome 4D, with both the $Q$ locus and $Q C p t$ on chromosome 4D increasing SPD due to the spelt wheat allele in this population (Manickavelu et al. 2011). It was still unclear whether QSpd.obu-4D and QCpt are identical QTL or not because of lack of common molecular markers used in Manickavelu et al. (2011) and this study. The QTL QGl.obu$1 A$ was detected only in the present study.

Regarding heading time, the LOD peak of QHt.obu-5A conferring later heading without vernalization due to the allele from CS was located on the gene specific marker from the Vrn-A1 locus. This result was consistent with the fact that CS has the vrn-Al allele (winter allele) on the $V r n-A 1$ locus (Yan et al. 2004). The location of QHt.obu-2B and
(A) Chromosome 2D

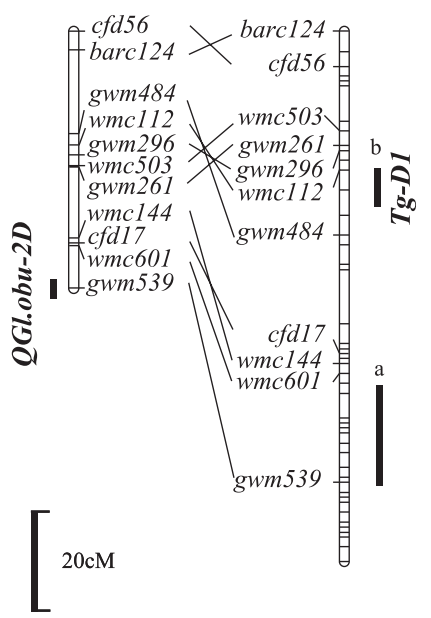

(B) Chromosome 4D

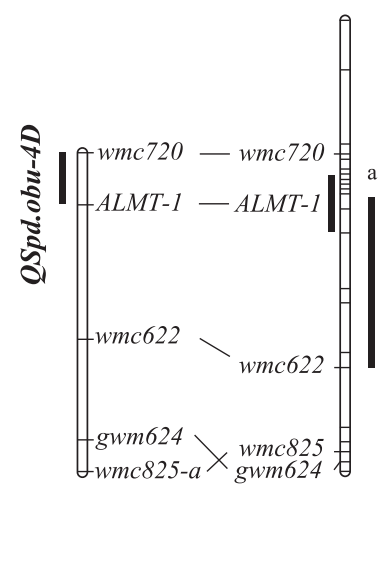

Fig. 4. Comparative maps of QTL locations on chromosomes 2D (A) and 4D (B). The linkage map in this study and the framework map based on Somers et al. (2004) are shown on the left and right, respectively. QTLs detected in this study are shown on the left linkage map, as boxes with two-LOD support intervals. The locations of reported QTLs or genes are shown on the right linkage map. a) Bars represent the regions including the LOD peaks of QTLs reported by Manickavelu et al. (2011). b) The location of $T g-D 1$ was reported by Dvorak et al. (2012). The location of ALMT-1 was reported by Ma et al. (2005). 


\section{Chromosome 2B}

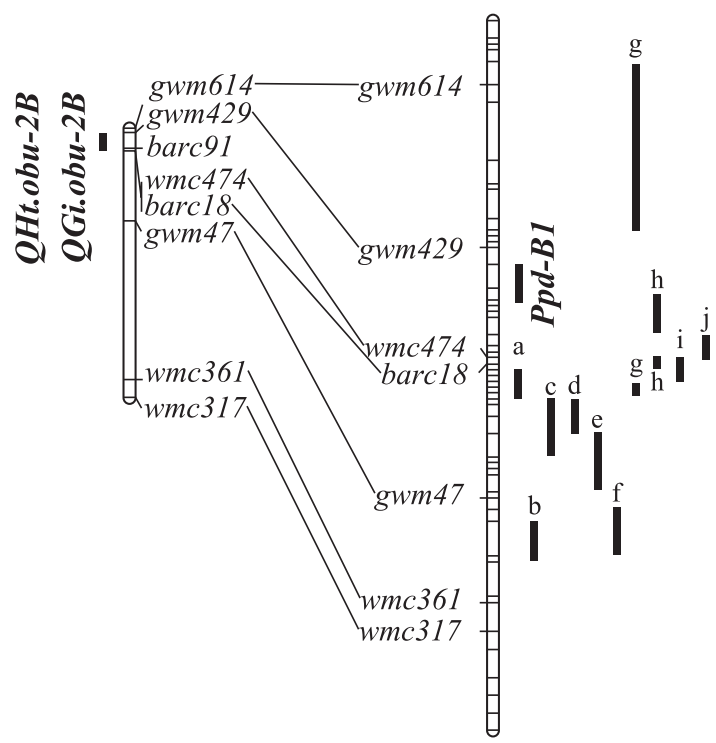

Fig. 5. Comparative maps of QTL locations for grain dormancy or PHS tolerance on chromosome $2 \mathrm{~B}$. The linkage map in this study and the framework map based on Somers et al. (2004) are shown on the left and right, respectively. QTLs detected in this study are shown on the left linkage map as boxes with two-LOD support intervals. The locations of the reported QTLs are shown on the right linkage map. Bars represent the regions including the LOD peaks of QTLs reported by a) and b) Chao et al. (2016); c) Kumar et al. (2015); d) Kulwal et al. (2004); e) Liu et al. (2008); f) Mohan et al. (2009); g) Singh et al. (2010); h) Somyong et al. (2014); i) Kumar et al. (2009); and j) Zhang et al. (2014). The location of Ppd-B1 was reported by Nguyen et al. (2013).

QHt.obu-7B were similar to the location of Ppd-B1 (Fig. 5, Nguyen et al. 2013) and Vrn-B3 (Yan et al. 2006), respectively. Although a QTL for heading time was also identified on chromosome $7 \mathrm{~B}$ in a bread wheat $\times$ spelt cross (Manickavelu et al. 2011), its location and effect were different from that of the QTL QHt.obu-7B (CS-derived allele conferred later heading). In bread wheat, a number of QTLs for grain dormancy or PHS tolerance exists near the QGi.obu-2B region (Fig. 5, Chao et al. 2016, Kulwal et al. 2004, Kumar et al. 2009, 2015, Liu et al. 2008, Mohan et al. 2009, Singh et al. 2010, Somyong et al. 2014, Zhang et al. 2014). Fine mapping by Somyong et al. (2014) revealed at least two QTLs in this region. In addition, the wheat $T a S d r$ gene, the ortholog of $O s S d r 4$ conferring grain dormancy in rice, was also present in this region (Zhang et al. 2014).

\section{Discussion}

The potential of spelt wheat as a genetic resource for the breeding of bread wheat has long been recognized (Campbell 1997). A wide range of phenotypic variation in spelt wheat for traits such as protein and mineral concentration of grains (Gomez-Becerra et al. 2010), aluminum tolerance (Raman et al. 2009), and various agronomic traits
(Miedaner and Longin 2016, Würschum et al. 2017) have been evaluated. However, studies identifying genes or QTLs associated with these traits in populations derived from crosses between spelt and bread wheat remain scarce (Liu et al. 2017, Manickavelu et al. 2011, Xie et al. 2015a, Zanetti et al. 2000). Identification of QTLs for valuable agronomic traits in spelt wheat is necessary to exploit its full potential for use in bread wheat breeding programs.

Spike morphology is a critical trait that affects the number of grains per spike and the grain morphology. In addition, spike morphology, including threshability, is taxonomically important because it is the most distinguishing trait between the bread and spelt wheat types. Therefore, understanding the genetic control of spike morphology is important not only for breeding, but also to clarify the origin of spelt wheat (Dvorak et al. 2012, Mac Key 1966). Transgressive segregations showing segregants with higher SPD than bread wheat were reported in several cross combinations between spelt and bread wheat (Dvorak et al. 2012, Manickavelu et al. 2011, Onishi et al. 2006, Xie et al. 2015a). Dvorak et al. (2012) indicated that spelt wheat had the $C$ allele for compact spikes on chromosome 2D despite the fact that spelt wheat had non-compact spikes, which supported the hypothesis that some spelt wheat, especially European spelt wheat, may have originated from hybridization between $T$. aestivum subsp. compactum carrying the $C$ allele and emmer wheat, which is a hulled or nonfree-threshing tetraploid wheat (Dvorak et al. 2012, Mac Key 1966). In contrast, QTLs increasing SPD, instead of the $C$ locus on chromosome 2D, due to alleles from spelt wheat were reported (Xie et al. 2015a). In the present study, a QTL for SPD showing stable effects under all the tested environmental conditions was detected on chromosome 4D (QSpd.obu-4D). In addition, the effect of $Q S p d . o b u-4 D$ was suppressed in the presence of the $q$ allele, implying that a cryptic variation, which can be revealed by crossing with bread wheat, has accumulated in spelt wheat and may reflect the different origins of spelt wheat.

The longer grain length observed in spelt wheat is one of the distinguishing characteristics in comparison to bread wheat (Campbell 1997, Gegas et al. 2010). A number of QTLs for grain length were detected in bread wheat $\times$ spelt crosses (Manickavelu et al. 2011, Xie et al. 2015b). Although the $T g-D 1$ gene on chromosome 2D was reported to have pleiotropic effects in increasing grain length (Okamoto et al. 2012), its utilization in bread wheat breeding programs has been difficult because $T g-D 1$ also confers the non-freethreshing trait. The QTLs QGl.obu-1A and QGl.obu-2D were independent of the QTLs for spike-related traits, thus highlighting their potential use in controlling grain length in bread wheat. The QTL QGl.obu-1A is a novel QTL obtained from the bread wheat $\times$ spelt cross although a few QTLs were previously reported at similar regions in bread wheat (Gegas et al. 2010). Further mapping of QGl.obu-1A is required for comparison with other QTLs found in bread wheat. 
Regarding the heading time, the QTL QHt.obu-7B, which had stable effects in environments with and without vernalization, was detected near the $V r n-B 3$ region on the chromosome 7B. The Vrn-B3 locus was cloned as the TaFT1 gene and a retrotransposon insertion was detected in the promoter region of the $\mathrm{Vrn}-\mathrm{B} 3$ allele from the cultivar HOPE, which conferred earlier heading time compared to the CS-derived allele (vrn-B3) (Yan et al. 2006). Previously, we identified a QTL in the Vrn-B3 region in RILs between Zenkouji-komugi $\times \mathrm{CS}$ and the Zenkouji-komugi-derived allele conferred earlier heading time compared to the CS-derived allele, despite the lack of retrotransposon insertions in Zenkouji-komugi (Cao et al. 2016). These results provided evidences that at least four alleles in a single or multiple QTL(s) could be located in the $V r n-B 3$ region, and that spelt wheat could serve as a novel genetic resource for QTL to modify heading time through breeding.

Grain dormancy of RU was lower than that of CS, although CS was revealed to be a weaker strain for grain dormancy in comparison to most Japanese wheat varieties (Chono et al. 2015). This result suggests that weak PHS tolerance may be a major obstacle in the utilization of spelt wheat as a genetic material for breeding in wet and humid regions such as Japan. To date, only a few studies have reported the evaluation of grain dormancy in spelt wheat (Liu et al. 2017, Ruzhitskaya and Borysova 2014) or PHS tolerance (Zanetti et al. 2000). In the present study, a QTL with stable effects $(Q G i . o b u-2 B)$ conferring weak dormancy due to the spelt wheat-derived allele was detected on chromosome 2B. In this region, as shown in Fig. 5, a number of QTLs, as well as some candidate genes affecting grain dormancy or PHS tolerance were reported. This indicates that the $Q G i . o b u-2 B$ region might be important for diversity of grain dormancy, not only among bread wheat varieties, but also between spelt and bread wheat. Fine mapping of QGi.obu- $2 B$ is needed for marker-assisted selection against the weak dormancy allele of spelt wheat. In addition, further elucidation of the genetic variation associated with grain dormancy in spelt wheat is important to determine its utility in breeding for diverse environmental conditions.

Spelt wheat was genetically differentiated from bread wheat based on DNA polymorphisms, such as single nucleotide polymorphisms (SNPs) in their genomes (Kippes et al. 2015). In addition, spelt wheat has specific taste and quality characteristics (Longin and Wurschum 2016). These results imply that there might be spelt wheat-specific genes or QTLs associated with phenotypes important for breeding. Further exploration and identification of useful genes or QTLs could accelerate the utilization of spelt wheat as a genetic resource in bread wheat breeding programs.

\section{Acknowledgments}

We thank Mayumi Tokui for her technical assistance. We also thank the anonymous reviewers for their valuable comments on earlier version of this paper. This work was partly supported by a grant from the Public Foundation of Elizabeth Arnold-Fuji.

\section{Literature Cited}

Asakura, N., N. Mori, C. Nakamura and I. Ohtsuka (2009) Genotyping of the $Q$ locus in wheat by a simple PCR-RFLP method. Genes Genet. Syst. 84: 233-237.

Campbell, K.G. (1997) Spelt: agronomy, genetics, and breeding. Plant Breed. Rev. 15: 187-213.

Cao, L., K. Hayashi, M. Tokui, M. Mori, H. Miura and K. Onishi (2016) Detection of QTLs for traits associated with pre-harvest sprouting resistance in bread wheat (Triticum aestivum L.). Breed. Sci. 66: 260-270.

Chao, S., E.Elias，D. Benscher，G. Ishikawa，Y.-F.Huang， M. Saito, T. Nakamura, S.Xu, J.Faris and M. Sorrells (2016) Genetic mapping of major-effect seed dormancy quantitative trait loci on chromosome 2B using recombinant substitution lines in tetraploid wheat. Crop Sci. 56: 1-14

Chono, M., H. Matsunaka, M. Seki, M.Fujita, C.Kiribuchi-Otobe, S. Oda, H. Kojima and S. Nakamura (2015) Molecular and genealogical analysis of grain dormancy in Japanese wheat varieties, with specific focus on MOTHER OF FT AND TFL1 on chromosome 3A. Breed. Sci. 65: 103-109.

Churchill, G.A. and R.W.Doerge (1994) Empirical threshold values for quantitative trait mapping. Genetics 138: 963-971.

Dvorak,J., K.R.Deal, M.C.Luo, F.M.You, K.von Borstel and H. Dehghani (2012) The origin of spelt and free-threshing hexaploid wheat. J. Hered. 103: 426-441.

Fu, D., P. Szucs, L. Yan, M. Helguera，J.S. Skinner，J.von Zitzewitz, P.M. Hayes and J.Dubcovsky (2005) Large deletions within the first intron in $V R N-1$ are associated with spring growth habit in barley and wheat. Mol. Genet. Genomics 273: 54-65.

Gegas, V.C., A. Nazari, S. Griffiths, J. Simmonds, L.Fish, S. Orford, L. Sayers, J.H. Doonan and J.W. Snape (2010) A genetic framework for grain size and shape variation in wheat. Plant Cell 22: 10461056 .

Gomez-Becerra, H.F., H. Erdem, A. Yazici, Y.Tutus, B.Torun, L. Ozturk and I.Cakmak (2010) Grain concentrations of protein and mineral nutrients in a large collection of spelt wheat grown under different environments. J. Cereal Sci. 52: 342-349.

Kamran,A., M. Iqbal and D. Spaner (2014) Flowering time in wheat (Triticum aestivum L.): a key factor for global adaptability. Euphytica 197: 1-26.

Kippes, N., J.M. Debernardi, H.A. Vasquez-Gross, B.A.Akpinar, H. Budak, K.Kato, S.Chao, E.Akhunov and J.Dubcovsky (2015) Identification of the VERNALIZATION 4 gene reveals the origin of spring growth habit in ancient wheats from South Asia. Proc. Natl. Acad. Sci. USA 112: E5401-E5410.

Kulwal, P.L., R. Singh, H.S. Balyan and P.K. Gupta (2004) Genetic basis of pre-harvest sprouting tolerance using single-locus and two-locus QTL analyses in bread wheat. Funct. Integr. Genomics 4: 94-101.

Kumar,A., J.Kumar, R. Singh, T. Garg, P. Chhuneja, H.S. Balyan and P.K. Gupta (2009) QTL analysis for grain colour and pre-harvest sprouting in bread wheat. Plant Sci. 177: 114-122.

Kumar, S., R.E.Knox, F.R.Clarke, C.J.Pozniak, R.M.DePauw, R.D. Cuthbert and S.Fox (2015) Maximizing the identification of QTL for pre-harvest sprouting resistance using seed dormancy measures in a white-grained hexaploid wheat population. Euphytica 205: $287-309$. 
Liu, S., S. Cai, R. Graybosch, C. Chen and G. Bai (2008) Quantitative trait loci for resistance to pre-harvest sprouting in US hard white winter wheat Rio Blanco. Theor. Appl. Genet. 117: 691-699.

Liu, Y., Y.Liu, Y.Zhou, C.Wight, Z.Pu, P.Qi, Q.Jiang, M.Deng, Z. Wang, Y. Wei et al. (2017) Conferring resistance to pre-harvest sprouting in durum wheat by a QTL identified in Triticum spelta. Euphytica 213: 19.

Longin, C.F. and T. Wurschum (2016) Back to the future-tapping into ancient grains for food diversity. Trends Plant Sci. 21: 731-737.

Ma, H.X., G.H. Bai, B.F. Carver and L.L. Zhou (2005) Molecular mapping of a quantitative trait locus for aluminum tolerance in wheat cultivar Atlas 66. Theor. Appl. Genet. 112: 51-57.

Mac Key, J. (1966) Species relationship in Triticum. In: Mac Key, J. (ed.) Proceedings of the 2nd International wheat genetics symposium; 1963, Mendelian Society of Lund for Scandinavian Association of Geneticists, Lund, Sweden, pp. 237-275.

Manickavelu,A., K. Kawaura, H.Imamura, M.Mori and Y.Ogihara (2011) Molecular mapping of quantitative trait loci for domestication traits and $\beta$-glucan content in a wheat recombinant inbred line population. Euphytica 177: 179-190.

Mares, D.J. and K. Mrva (2014) Wheat grain preharvest sprouting and late maturity alpha-amylase. Planta 240: 1167-1178.

Matsuoka,Y. (2011) Evolution of polyploid Triticum wheats under cultivation: the role of domestication, natural hybridization and allopolyploid speciation in their diversification. Plant Cell Physiol. 52: 750-764.

Miedaner, T. and C.F.H.Longin (2016) Neglected cereals: From ancient grains to superfood. Agrimedia, Clenze, Germany, p. 157.

Mohan,A., P.Kulwal, R. Singh, V.Kumar, R.R.Mir, J.Kumar, M. Prasad, H.S.Balyan and P.K.Gupta (2009) Genome-wide QTL analysis for pre-harvest sprouting tolerance in bread wheat. Euphytica 168: 319-329.

Monna, L., H.X. Lin, S. Kojima, T. Sasaki and M. Yano (2002) Genetic dissection of a genomic region for a quantitative trait locus, $H d 3$, into two loci, $H d 3 a$ and $H d 3 b$, controlling heading date in rice. Theor. Appl. Genet. 104: 772-778.

Nguyen,A.T., J.C.M.Iehisa, N. Mizuno, M.Nitta, S.Nasuda and S. Takumi (2013) Differential contribution of two Ppd-1 homoeoalleles to early-flowering phenotype in Nepalese and Japanese varieties of common wheat. Breed. Sci. 63: 374-383.

Okamoto, Y., T. Kajimura, T.M.Ikeda and S. Takumi (2012) Evidence from principal component analysis for improvement of grain shape- and spikelet morphology-related traits after hexaploid wheat speciation. Genes Genet. Syst. 87: 299-310.

Onishi, I., A. Hongo, T. Sasakuma, T. Kawahara, K. Kato and H. Miura (2006) Variation and segregation for rachis fragility in spelt wheat, Triticum spelta L. Genet. Resour. Crop Evol. 53: 985-992.

Raman,H., R. Rahman, D.Luckett, R. Raman, F.Bekes, L.Láng and Z. Bedo (2009) Characterisation of genetic variation for aluminium resistance and polyphenol oxidase activity in genebank accessions of spelt wheat. Breed. Sci. 59: 373-381.

Ruzhitskaya, O. and O.Borysova (2014) Germination and quality of spelt seeds under southwestern Ukraine condition. Genetics Plant Physiol. 4: 216-224.

Sasaki, T., P.R. Ryan, E. Delhaize, D.M.Hebb, Y. Ogihara, K. Kawaura, K. Noda, T.Kojima, A.Toyoda, H. Matsumoto et al. (2006) Sequence upstream of the wheat (Triticum aestivum L.) ALMT1 gene and its relationship to aluminum resistance. Plant Cell Physiol. 47: 1343-1354.

Simons, K.J., J.P. Fellers, H.N. Trick, Z.Zhang, Y.S. Tai, B.S. Gill and J.D. Faris (2006) Molecular characterization of the major wheat domestication gene $Q$. Genetics 172: 547-555.

Singh, R., M. Matus-Cádiz, M. Båga, P. Hucl and R.N. Chibbar (2010) Identification of genomic regions associated with seed dormancy in white-grained wheat. Euphytica 174: 391-408.

Somers, D.J., P.Isaac and K.Edwards (2004) A high-density microsatellite consensus map for bread wheat (Triticum aestivum L.). Theor. Appl. Genet. 109: 1105-1114.

Somyong, S., G. Ishikawa， J.D. Munkvold，J.Tanaka，D. Benscher, Y.G.Cho and M.E. Sorrells (2014) Fine mapping of a preharvest sprouting QTL interval on chromosome 2B in white wheat. Theor. Appl. Genet. 127: 1843-1855.

Tanabata, T., T. Shibaya, K.Hori, K.Ebana and M.Yano (2012) SmartGrain: high-throughput phenotyping software for measuring seed shape through image analysis. Plant Physiol. 160: 1871-1880.

van Ooijen, J.W. (2002) MapQTL ${ }^{\circledR}$ 6, Software for the mapping of quantitative trait loci in experimental populations of diploid species. Kyazma B.V., Wageningen, The Netherlands.

van Ooijen, J.W. (2006) JoinMap ${ }^{\circledR}$ 4, Software for the calculation of genetic linkage maps in experimental population. Kyazma B.V., Wageningen, The Netherlands.

Würschum, T., W.L. Leiser and C.F.H. Longin (2017) Molecular genetic characterization and association mapping in spelt wheat. Plant Breed. 136: 214-223.

Xie, Q., S. Mayes and D.L. Sparkes (2015a) Spelt as a genetic resource for yield component improvement in bread wheat. Crop Sci. 55: 2753-2765.

Xie, Q., S. Mayes and D.L. Sparkes (2015b) Carpel size, grain filling, and morphology determine individual grain weight in wheat. J. Exp. Bot. 66: 6715-6730.

Yan, L., M.Helguera, K.Kato, S.Fukuyama, J.Sherman and J. Dubcovsky (2004) Allelic variation at the $V R N-1$ promoter region in polyploid wheat. Theor. Appl. Genet. 109: 1677-1686.

Yan, L., D. Fu, C. Li, A. Blechl, G. Tranquilli, M. Bonafede, A. Sanchez, M. Valarik, S. Yasuda and J. Dubcovsky (2006) The wheat and barley vernalization gene VRN3 is an orthologue of FT. Proc. Natl. Acad. Sci. USA 103: 19581-19586.

Zanetti, S., M. Winzeler, M. Keller, B. Keller and M. Messmer (2000) Genetic analysis of pre-harvest sprouting resistance in a wheat $\times$ spelt cross. Crop Sci. 40: 1406-1417.

Zhang, Y., X. Miao, X. Xia and Z.He (2014) Cloning of seed dormancy genes $(T a S d r)$ associated with tolerance to pre-harvest sprouting in common wheat and development of a functional marker. Theor. Appl. Genet. 127: 855-866. 